\title{
Quality Indicators of Transanal Total Mesorectal Excision (TaTME) for Rectal Cancer
}

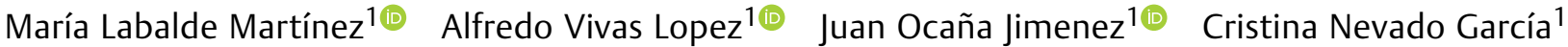

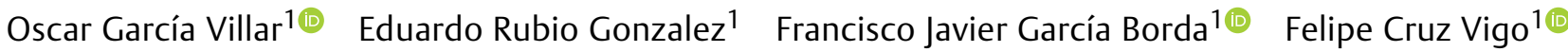 \\ Eduardo Ferrero Herrero ${ }^{10}$ \\ ${ }^{1}$ Unit of Colorectal Surgery, Department of General and Digestive \\ Surgery and Abdominal Organ Transplantation, Hospital \\ Universitario 12 de Octubre, Madrid, Spain \\ J Coloproctol 2021;41(4):411-418

\begin{abstract}
Address for correspondence Maria Labalde Martínez, MD, PhD, Unidad de Cirugía Colorrectal, Servicio de Cirugía General, Aparato Digestivo y Trasplante de Órganos Abdominales, Hospital Universitario 12 de Octubre, Avenida de Córdoba s/n, Madrid - 28041, Spain (e-mail: mlabaldemartinez@yahoo.es).
\end{abstract}

\begin{abstract}
Keywords

- rectal cancer

- transanal total mesorectal excision rectal cancer surgery

- TaTME

- quality indicators of rectal cancer surgery

Introduction Transanal total mesorectal excision (TaTME) has revolutionized the surgical techniques for lower-third rectal cancer. The aim of the present study was to analyze the outcomes of quality indicators of TaTME for rectal cancer compared with laparoscopic TME (LaTME).

Methods A cohort prospective study with 50 ( 14 female and 36 male) patients, with a mean age of 67 (range: 55.75 to 75.25 ) years, who underwent surgery for rectal cancer. In total, 20 patients underwent TaTME, and 30, LaTME. Every TaTME procedure was performed by experienced colorectal surgeons. The sample was divided into two groups (TaTME and LaTME), and the quality indicators of the surgery for rectal cancer were analyzed.

Results There were no statistically significant differences regarding the patients and the main characteristics of the tumor (age, gender, American Society of Anesthesiologists [ASA] score, body mass index [BMI], tumoral stage, neoadjuvant therapy, and distance from the tumor to the external anal margin) between the two groups. The rates of: postoperative morbidity (TaTME: $35 \%$; LaTME: $30 \% ; p=0.763$ ); mortality ( $0 \%$ ); anastomotic leak (TaTME: 10\%; LaTME: $13 \% ; p=0.722$ ); wound infection (TaTME: 0\%; LaTME: $3.3 \% ; p=0.409$ ); reoperation (TaTME: $5 \%$; LaTME: $6.6 \% ; p=0.808$ ); and readmission (TaTME: $5 \%$; LaTME: $0 \% ; p=0.400$ ), as well as the length of the hospital stay (TaTME: 13.5 days; LaTME: 11 days; $p=0.538$ ), were similar in both groups. There were no statistically significant differences in the rates of positive circumferential resection margin (TaTME: 5\%; LaTME: 3.3\%; $p=0.989$ ) and positive distal resection margin (TaTME: $0 \%$; LaTME: 3.3\%; $p=0.400$ ), the completeness of the TME (TaTME: 100\%; LaTME: 100\%), and the number of lymph nodes harvested (TaTME: 15; LaTME: $15.5 ; p=0.882$ ) between two groups.

Conclusion Transanal total mesorectal excision is a safe and feasible surgical procedure for middle/lower-third rectal cancer.
\end{abstract}

received

February 20, 2021

accepted after revision

June 15, 2021
DOI https://doi.org/

$10.1055 / \mathrm{s}-0041-1736640$.

ISSN 2237-9363. (c) 2021. Sociedade Brasileira de Coloproctologia. All rights reserved.

This is an open access article published by Thieme under the terms of the Creative Commons Attribution-NonDerivative-NonCommercial-License, permitting copying and reproduction so long as the original work is given appropriate credit. Contents may not be used for commercial purposes, or adapted, remixed, transformed or built upon. (https://creativecommons.org/ licenses/by-nc-nd/4.0/)

Thieme Revinter Publicações Ltda., Rua do Matoso 170, Rio de Janeiro, RJ, CEP 20270-135, Brazil 


\section{Introduction}

Rectal cancer is the seventh most common cancer worldwide. ${ }^{1}$ In a 2017 study $^{2}$ regarding six European countries, the 5 -year overall survival rate for rectal cancer ranged from $55 \%$ to $62 \%$. This rate tends to improve due to new challenges, including the adoption of multidisciplinar management and guidelines founded on evidence based-medicine, and the development of surgical techniques. ${ }^{2}$ Total mesorectal excision (TME), which was described by Heald et al. $^{3}$ in 1982, and an adequate circumferential resection margin (CRM) have strongly reduced the recurrence rate. In two randomized controlled trials, ${ }^{4,5}$ the authors could not demonstrate the non-inferiority of laparoscopic TME (LaTME) over open TME regarding the pathological outcomes, and they recommended the inclusion of additional approaches to abdominal laparoscopy in low rectal cancer with risk factors for intraoperative difficulties.

The development of transanal minimally-invasive surgery using transanal platforms and laparoscopic instruments has facilitated the development of rectal surgery towards the transanal TME (TaTME) procedure. First described by Sylla et al. ${ }^{6}$ in 2010, TaTME enables a better visualization of the dissection plans, and seems to facilitate the complete mobilization of the mesorectum and the distal transection of rectum. Therefore, these technical advantages could increase the number of sphincter-saving procedures and improve the outcomes of rectal cancer surgery.

The aim of the present study is to analyze and compare the indicators of quality of rectal cancer surgery of a series of patients with lower- and middle-third rectal cancer who underwent TaTME or LaTME with coloanal anastomosis.

\section{Material and Methods}

From June 2017 to July 2018, the rectal-cancer patients who underwent TaTME or LaTME with colorectal anastomosis in the Coloproctology Unit of Hospital Universitario 12 de Octubre, in Madrid, Spain, were included in the present study. These procedures were approved by the Ethics Committee of the hospital.

The diagnosis of rectal cancer was established by histological analysis of the tumor biopsy obtained at colonoscopy. The preopeative evaluation included: complete history and physical examination with digital rectal exam, rigid proctoscopy and full colonoscopy, the level of carcinoembryonic antigen (CEA), and thoraco-abdominal computed tomography $(\mathrm{CT})$ and magnetic resonance imaging (MRI). The definition of the location of the rectal cancer was based on preoperative MRIs according to the distance from the anorectal junction to the distal margin of the tumour: up to $4 \mathrm{~cm}$ from the anorectal junction - lower third of the rectum; $4 \mathrm{~cm}$ to $8 \mathrm{~cm}$ - middle-third of the rectum; and $8 \mathrm{~cm}$ to $12 \mathrm{~cm}$ upper third of the rectum. Patients with cancer located in the upper third of the rectum were excluded. Rectal cancer was staged according to the 8th edition of the tumor, node, metastasis (TNM) classification. ${ }^{8}$ Cases of diagnosed rectal cancer with a complete preoperative evaluation to stage the tumor were discussed by a multidisciplinary oncological team, including a radiologist, a radiation oncologist, surgeons, and a pathologist to develop appropriate individualized treatment plans according to evidence-based guidelines. The indication for neoadjuvant chemoradiotherapy was in accordance with the European Society for Medical Oncology (ESMO) guidelines. ${ }^{8}$ Therefore, patients with tumors in stages T3-T4N0 or T1-T4N1-N2 were eligible for neoadjuvant chemoradiotherapy. Restaging of the rectal cancer was performed by lower abdomen MRI and thoracoabdominal CT about six to eight weeks after the end of the chemoradiotherapy course.

The inclusion criteria were as follows: ${ }^{1}$ histologicallyproven rectal adenocarcinomas, ${ }^{2}$ and tumors located $\geq 8 \mathrm{~cm}$ from the anorectal junction determined by MRI. And the exclusion criteria were: ${ }^{1}$ inability to perform sphincter preservation, ${ }^{2}$ cases of recurrent cancer, ${ }^{3}$ and cases of emergency surgery.

Every surgical intervention was performed by experienced surgeons specialized in laparoscopic colorectal surgery. The TaTME procedure was performed in lower- and middle-third rectal cancer patients according to the surgeon's discretion and experience. Threatened CRM was considered a contraindication to TaTME in the present study.

Data regarding the patients were collected from a prospectively-generated database, and they included gender, age, body mass index (BMI), the score on the American Society of Anesthesiologists (ASA) Physical Status Classification System, tumor location, preoperative staging, the level of CEA, neoadjuvant chemoradiotherapy, pathological features of the specimens (staging, number of lymph nodes harvested, TME quality, tumor regression grade), and length of hospital stay.

The following quality indicators of the surgery for rectal cancer were considered: rate of positive CRM, rate of positive DRM, rate of complete TME, rate of postoperative morbidity, mortality, rate of anastomotic leak, rate of abdominal wound infection, and the reoperation and readmission rates.

\section{Surgical Technique}

Each patient underwent preoperative bowel preparation, and potential stoma (colostomy and ileostomy) sites were marked by an ostomal therapist the day before surgery. Venous thromboembolism prophylaxis and intravenous antibiotic prophylaxis were performed according to the protocols of our hospital. A rectal enema with diluted iodine solution is performed immediately before surgery.

The TaTME procedure was performed under general anesthesia in the Trendelemburg lithotomy position by two teams of experienced surgeons working simultaneously. During the transanal stage of the procedure, an anal retractor (Lonestar, CooperSurgical, Inc., Trumbull, CT, United States) was used to expose the dentate line, and a pneumorectum was created using the GelPOINT Path Transanal Access Platform (Applied Medical Resources Corporation, Rancho Santa Margarita, CA, Unitred States) after the clamping of the sigmoid colon by the abdominal team. The rectal lumen was closed with a purse-string suture distal to the tumor. A washout of the distal rectum stump 
with a diluted povidone-iodine solution was needed. The line of the rectotomy was marked by tattooing the rectal mucosa circumferentially with a monopolar diathermy probe, and a full-thickness, perpendicular transection of the rectal wall was performed. The TME was completed circumferentially from top to bottom starting with the posterior and anterior planes followed by lateral ones. During the abdominal stage of the procedure, a pneumoperitoneum was created with a Verres needle, and 4 laparoscopic ports (2 measuring $12 \mathrm{~mm}$ and $11 \mathrm{~mm}$, and 2 measuring $5 \mathrm{~mm}$ ) were inserted. Abdominal exploration was performed to exclude any undiagnosed pathologies. Complete mobilization of the splenic flexure of the colon was performed if needed. A high ligation of the inferior mesenteric artery was performed, and the inferior mesenteric vein was ligated at the level of the inferior border of the pancreas. The rectal dissection was continued from the posterior plane circumferentially to the anterior plane. The transanal and abdominal teams worked in coordination to achieve better visualization and to complete the dissection. Both planes were connected at the anterior midline, and the peritoneal reflection was completely opened. The specimen was extracted transanally without trauma. In cases of bulky tumors or narrow pelvis, the specimen was extracted transabdominally through a Pfannestiel incision. The bowel was divided extracorporeally. The anvil of an EEA stapler (Medtronic, Minneapolis, MN, United States) was inserted and secured inside the proximal colon. A full-thickness purse-string suture of the open rectal stump is required, tied around a drain inserted through the central opening rectal cuff. The distal end of the drain was attached to the spike of a circular stapler. The laparoscopic team removed the drain and connected the anvil and the spike of the stapler. A side-to-end colorectal anastomosis was performed using a standard EEA stapler (with a that diameter varied from $29 \mathrm{~mm}$ to $33 \mathrm{~mm}$ ) under laparoscopic vision. The anastomosis was tested by transanal air insufflation. A protective ileostomy was performed on the right lower quadrant, if necessary. A drain was placed in the pelvis. The LaTME technique was performed following the same procedures, including those for the abdominal stage of TaME. ${ }^{7}$

\section{Histopathological Examination}

The specimen was evaluated by pathologists specialized in rectal cancer. The tumors were staged according to the 8th edition of the TNM classification. ${ }^{8}$ The CRM was defined as positive when there was a distance shorter than $1 \mathrm{~mm}$ between the deepest cancer invasion and the surgical resection margin. The DRM was considered positive if it was shorter than $1 \mathrm{~cm}$. The completeness of the TME was standardized according to the integrity of the mesorectum delineated by Nagtegaal et al. ${ }^{9}$ The tumor response to neoadjuvant chemoradiotherapy was defined by the modified Ryan tumor regression grade (TRG) system. ${ }^{10}$

\section{Quality Indicators for Rectal Cancer Surgery}

Postoperative morbidity was defined as any complications ocurring until the 30th postoperative day, which were graded according to the Clavien-Dindo classification. ${ }^{11}$ Anastomotic leakage included both symptomatic and asymptomatic with radiological features leaks. Reoperation and readmission included 30-day of discharge. Mortality was defined as death occurring in the hospital or within 30 days of discharge. ${ }^{12}$

\section{Statistical Analysis}

The quantitative data were reported as intercuartile ranges, and the qualitative data were reported as numbers and percentages of patients. The Shapiro-Wilk test was used to check the normal distribution of the data. Dichotomous and categorical values were analyzed using the Chi-squared $\left(\chi^{2}\right)$ or Fisher exact tests as appropriate. The continuous variables were compared using the independent Student $t$-test for normally distributed data, and the Mann-Whitney test, for non-normally distributed data. All variables that were significant in the univariate analyses and those considered clinically relevant were included in a multivariate stepwise regression analysis to determine which variables were statistically significant independent risk factors. Differences were considered statistically significant when $p<0.05$. All analyses were performed using the Statistical Package for the Social Scineces (IBM SPSS Statistics for Windows, IBM Corp., Armonk, NY, United States) software, version 23.0.

\section{Results}

\section{Patient Characteristics and Pathological Features of the Tumor}

A total of 50 patients with lower- and middle-third rectal cancer were treated by surgery with curative intent, 20 of whom underwent TaTME. The sample as composed of 36 (72\%) male patients and $14(28 \%)$ female patients with a mean age of 67 (range: 55.75 to 75.25 ) years. The mean distance of the tumor from the anal verge measured by MRI was of $7 \mathrm{~cm}$ (range: $4 \mathrm{~cm}$ to $8 \mathrm{~cm}$ ). At diagnosis, $16(32 \%)$ patients were staged as cT1-T2, and $24(48 \%)$, as $\mathrm{cN}+$. A total of 24 (48\%) patients underwent neoadjuvant radiochemotherapy. The pathological examination revealed 21 cases of locally-advanced tumors (pT3-4), and 6 of them presented complete response to neoadjuvant therapy (grades 0 and 1 in the modified Ryan TRG system).The main characteristics of the patients and pathological features of the tumor are presented in -Table 1. The data shows there were no statistically significant differences in these results between the two groups.

\section{Quality Indicators of Rectal Cancer Surgery}

The quality indicators of rectal cancer surgery are presented in - Table 2. The rate of postoperative morbidity was of $32 \%$ (TaTME: 35\%; LaTME: 30\%; $p=0,763$ ). There were 13 patients with minor complications (grades I or II in the Clavien-Dindo classification), 6 in the TaTME group ( 3 cases of ileus, 1 case of pulmonary embolism, and 2 cases of anastomotic leak) and 7 in the LaTME group ( 1 case of wound infection, 2 episodes of bleeding, 3 cases of anastomotic leak, and 1 case of leg pain), and there were 3 patients with grade- 
414 Quality Indicators of Transanal Total Mesorectal Excision for Rectal Cancer Labalde Martínez et al.

Table 1 Patient characteristics and pathological features of the tumors

\begin{tabular}{|c|c|c|c|c|}
\hline & $\begin{array}{l}\text { Total } \\
(n=50)\end{array}$ & $\begin{array}{l}\text { TaTME } \\
(n=20)\end{array}$ & $\begin{array}{l}\text { LaTME } \\
(n=30)\end{array}$ & $p$-value \\
\hline \multicolumn{5}{|l|}{ Gender } \\
\hline Male & $36(72 \%)$ & $13(65 \%)$ & $23(76.7 \%)$ & \multirow[t]{2}{*}{0.363} \\
\hline Female & $14(28 \%)$ & 7 (35\%) & $7(23.3 \%)$ & \\
\hline Age (years) & $67(55.7-75.2)$ & $66.5(55.5-66.5)$ & $67(55.7-73)$ & 0.977 \\
\hline \multicolumn{5}{|l|}{ ASA score } \\
\hline 1 & $2(4 \%)$ & $2(10 \%)$ & 0 & \multirow[t]{4}{*}{0.086} \\
\hline II & $20(40 \%)$ & $5(25 \%)$ & $15(50 \%)$ & \\
\hline III & 27 (54\%) & $12(60 \%)$ & $15(50 \%)$ & \\
\hline IV & $1(2 \%)$ & $1(5 \%)$ & 0 & \\
\hline BMI $\left(\mathrm{kg} / \mathrm{m}^{2}\right)$ & $25.3(23.7-28.1)$ & $25.7(22.5-28.6)$ & $25.1(23.7-28.1)$ & 0.488 \\
\hline \multicolumn{5}{|l|}{ Tumor location } \\
\hline Lower third of the rectum & $15(30 \%)$ & 7 (35\%) & $8(26.6 \%)$ & \multirow[t]{2}{*}{0.529} \\
\hline Middle third of the rectum & $35(70 \%)$ & $13(65 \%)$ & $22(73.3 \%)$ & \\
\hline Distance of the tumor from the anal verge $(\mathrm{cm})$ & $7(4-8)$ & $7(4-7)$ & $6(4-7)$ & 0.273 \\
\hline \multicolumn{5}{|l|}{ Preoperative stage } \\
\hline cT1 & $6(12 \%)$ & $1(5 \%)$ & $5(16.7 \%)$ & 0.380 \\
\hline cT2 & $10(20 \%)$ & $6(30 \%)$ & $4(13.3 \%)$ & 0.729 \\
\hline cT3 & $26(52 \%)$ & $10(50 \%)$ & $16(53.3 \%)$ & \\
\hline cT4 & $8(16 \%)$ & $3(15 \%)$ & $5(16.7 \%)$ & \\
\hline $\mathrm{cN}+$ & $24(48 \%)$ & $9(45 \%)$ & $15(50 \%)$ & \\
\hline CEA (ng/mL) & $3.7(1.8-6.5)$ & $4.7(1.44-7.35)$ & $2.74(1.86-6.3)$ & 0.187 \\
\hline Neoadjuvant therapy & $26(52 \%)$ & $11(55 \%)$ & $15(50 \%)$ & 0.726 \\
\hline \multicolumn{5}{|l|}{ Pathological tumor stage } \\
\hline pT3-4 & $21(42 \%)$ & $9(45 \%)$ & $13(43.3 \%)$ & 0.880 \\
\hline $\mathrm{N}+$ & $11(22 \%)$ & $5(25 \%)$ & $6(20 \%)$ & 0.736 \\
\hline \multicolumn{5}{|l|}{ Ryan tumor regression grade system } \\
\hline Grade 0 & $2(4 \%)$ & $1(5 \%)$ & $1(3.3 \%)$ & \multirow[t]{6}{*}{0.384} \\
\hline Grade 1 & $4(8 \%)$ & $2(10 \%)$ & $2(6.6 \%)$ & \\
\hline Grade 2 & $6(12 \%)$ & $1(5 \%)$ & $5(16.6 \%)$ & \\
\hline Grade 3 & $11(22 \%)$ & $6(30 \%)$ & $5(16.6 \%)$ & \\
\hline Grade 4 & $2(4 \%)$ & 0 & $2(6.6 \%)$ & \\
\hline Grade 5 & $1(2 \%)$ & $1(5 \%)$ & 0 & \\
\hline
\end{tabular}

Abbreviations: ASA, American Society of Anesthesiologists; BMI, body mass index; CEA, carcinoembryonic antigen; LaTME, laparoscopic total mesorectal excision; TaTME, transanal total mesorectal excision.

III complications in the Clavien-Dindo classification, 1 in the TaTME group (hemorrhage), and 2 in the LaTME group (1 case of hemorrage and 1 case of anastomotic leak). The rate of anastomotic leak was of 12\% (TaTME: 10\%; LaTME: $13 \%$; $p=0,722$ ): 5 cases were treated with intravenous antibiotics and parenteral nutrition, and 1 case needed reintervention by the Hartmann procedure. Two patients were reoperated on for hemorrhage and anastomotic leak in the LaTME group, and one, for anastomotic leak in the TaTME group. One patient who underwent TaTME was readmitted because of pelvic collection and pulmonary embolism, and one in the LaTME died because of sepsis.

The pathological examination revealed that the whole sample had complete or nearly complete TME. There were two cases (one in the TaTME group and one in the LaTME group) with positive CRM, and one case with positive DRM in the LaTME group.

The multiple logistic regression analysis, including the statistically significant variables in the univariate analysis and those considered clinically relevant, showed that TaTME 
Table 2 Quality indicators of rectal cancer surgery

\begin{tabular}{|l|l|l|l|l|}
\hline & $\begin{array}{l}\text { Total } \\
(N=50)\end{array}$ & $\begin{array}{l}\text { TaTME } \\
(N=20)\end{array}$ & $\begin{array}{l}\text { LaTME } \\
(N=30)\end{array}$ & -value \\
\hline Operative time & $290(225-300)$ & $367(292-420)$ & $240(196-240)$ & $0.001^{*}$ \\
\hline Rate of postoperative morbidity & $16(32 \%)$ & $7(35 \%)$ & $9(30 \%)$ & 0.763 \\
\hline Clavien-Dindo classification & $13(26 \%)$ & $6(30 \%)$ & $7(23.3 \%)$ & 0.808 \\
\hline I-II & $3(6 \%)$ & $1(5 \%)$ & $2(6.6 \%)$ & \\
\hline III-IV & $6(12 \%)$ & $2(10 \%)$ & $4(13 \%)$ & 0.722 \\
\hline Rate of anastomotic leak & $1(2 \%)$ & 0 & $1(3.3 \%)$ & $0 . .409$ \\
\hline Rate of abdominal wound infection & $11(7-17)$ & $13,5(8.2-17)$ & $11(7-17)$ & 0.538 \\
\hline Length of hospital stay (days) & $3(6 \%)$ & $1(5 \%)$ & $2(6.6 \%)$ & 0.808 \\
\hline Reoperation rate & $1(2 \%)$ & $1(5 \%)$ & 0 & 0.400 \\
\hline Readmission rate & $1(2 \%)$ & 0 & $1(3.3 \%)$ & 0.400 \\
\hline Mortality & $2(4 \%)$ & $1(5 \%)$ & $1(3.3 \%)$ & 0.989 \\
\hline Positive CRM & $1(2 \%)$ & 0 & $1(3.3 \%)$ & 0.400 \\
\hline Positive DRM & $50(100 \%)$ & $20(100 \%)$ & $30(100 \%)$ & - \\
\hline Complete-nearly complete TME & $15(12-21.2)$ & $15(12-20.5)$ & $15.5(10.7-22)$ & 0.882 \\
\hline Number of lymph nodes harvested & & & \\
\hline
\end{tabular}

Abbreviations: CRM, circumferential resection margin; DRM, distal resection margin; LaTME, laparoscopic total mesorectal excision; TaTME, transanal total mesorectal excision; TME, total mesorectal excision.

Note: *Statistically significant differences.

was not independently associated with the morbidity rate, positive CRM and positive DRM ( - Table 3 ).

\section{Discussion}

The results of the present study suggest that TaTME is a safe and feasible surgical procedure for lower-third rectal cancer, because the indicators of quality for rectal cancer surgery were similar after the TaTME and LaTME procedures in the present series. There were no significant differences between the two study groups in terms of the rate of postoperative morbidity rate, mortality, the rates of reoperation and readmission rate, and the pathological features, such as the rates of positive CRM and DRM and the completeness of TME. The advantages that TaTME offers related to the improved visualization of the CRM and DRM and dissection planes could make this procedure adequate for middle-/lower-third rectal cancer. Since the TaTME was described, many groups of researchers have published the outcomes of their groups of patients with the procedure.
During LaTME, the use of instruments is limited because they are fixed at the entry point of the trocar in the abdominal wall, and, in cases of patients with a narrow pelvis, it is particularly difficult to reach the lower third of the rectum deep in the pelvis and to perform a single shot during transection. ${ }^{13,14}$ The TaTME procedure could reduce the effects on the surgical outcomes of the unfavourable conditions for laparoscopic rectal surgery, like obesity, the male gender, neoadjuvant therapy, and narrow pelvis. At least, we published these adverse conditions for laTME did not get worse the morbidity rate, conversion rate, operative time and pathological outcomes in patients underwent TaTME with adverse conditions for LaTME. ${ }^{13}$ Indeed, the Robotic Versus Laparoscopic Resection for Rectal Cancer (ROLARR) randomized trial ${ }^{15}$ failed to demonstrate significant benefits of robotic surgery over LaTME in relation to postoperative morbidity and pathological outcomes. Thus, TaTME would be an alternative to make TME for lower-third rectal cancer easier in unfavourable cases.

Table 3 Multivariate analysis regarding the quality indicators of rectal cancer surgery and TaTME

\begin{tabular}{|l|l|l|l|l|}
\hline & $\beta$ coefficient & $p$-value & Odds ratio & $\begin{array}{l}\text { 95\% confidence } \\
\text { interval }\end{array}$ \\
\hline Morbidity & 0.228 & 0.711 & 1.256 & $0.376-4.196$ \\
\hline Positive circumferential resection margin & 0.423 & 0.770 & 1.526 & $0.090-25.904$ \\
\hline Positive distal resection margin & -0.405 & 0.160 & 0.667 & $0.001-36.923$ \\
\hline
\end{tabular}


The TaTME procedure enables the dissection of the mesorectum through the anus using a transanal endoscopic platform to create a pneumorectum. This approach improves the visualization of the least accessible portion of the rectum, and could be advantageous in several surgical procedures. The main indication of TaTME is in the treatment of malignant tumors located in the middle and lower thirds of the rectum, as we have done in the present study. The TaTME procedure could also be indicated in cases of inflammatory bowel disease requiring proctectomy, reversal of the Hartmann procedure in cases of failure to progress during conventional abdominal surgery, familiar adenomatous polyposis, transanal completion of TME after local excision of early tumors, and rectal strictures. The possible contraindications to TaTME are locally-advanced rectal tumors with threatened CRM, prostate infiltration, or sphincter involvement demonstrated in preoperative study because of technical difficulties in in preoperative study. ${ }^{16,17}$ We included cT4 tumors in both groups ( 3 cases in the TaTME group and 5 in the LaTME group), and there were no statistically significant differences between the groups in terms of preoperative and pathological tumor stages. However, we did not report the outcomes for this specific group. Other authors also include locally-advanced rectal tumors underwent TaTME as we made with optimal pathological and clinical outcomes suggesting experienced surgeons in TaTME could successfully perform it in these cases. ${ }^{18-21}$ Therefore, we do not recommend TaTME in patients with locally-advanced rectal tumors during the learning curve for the TaTME procedure. ${ }^{22}$

The rate of postoperative morbidity in the present series was of $32 \%$, and there were no statistically significant differences between the TaTME (35\%) and LaTME (30\%) groups $(p=0.763)$. In relation to the Clavien-Dindo classification, there was a rate of $6.6 \%$ of grades III-IV postoperative complications (TaTME: 5\%; LaTME: $6.6 \% ; p=0.808$ ). There are two randomized trials ${ }^{23,24}$ still in progress, but the results of two other retrospective studies ${ }^{25,26}$ and of the International TaTME Registry, 27,28,32 have already been reported. As part of the International TaTME Registry, Penna et al. ${ }^{27}$ analyzed data from 66 surgical centres in 23 different countries regarding 720 patients with benign and malignant diseases treated by TaTME; they reported that the rate of postoperative morbidity at 30 days was of $32 \%$, including $10 \%$ of cases of grade-III and $1 \%$ of cases of grade-IV complications in the Clavien-Dindo classification. These results were similar to those reported in the present study. Two meta-analysis $^{25,26}$ including retrospective studies compared perioperative outcomes after TaTME and LaTME, and reported that the TaTME group had a lower rate of postoperative complications, ranging from $27 \%$ to $35 \%$, compared to the LaTME group.

Another study ${ }^{28}$ from the International TaTME Registry, on 1,594 patients who underwent surgery in 107 hospitals, reported an overall rate of anastomotic failure of $15.7 \%$, including $7.8 \%$ of cases of early leak, $2 \%$ of cases of delayed leak, $4.7 \%$ of cases of pelvic abscess, $0.8 \%$ of cases of anastomotic failure, $0.9 \%$ of cases of chronic sinus, and $3.6 \%$ of cases of anastomotic stricture. ${ }^{28}$ In the present study, there was a rate of $12 \%$ of anastomotic leaks diagnosed within 30 days of the primary resection (TaTME: $10 \%$; LaTME: $13 \% ; p=0.722$ ). Only one patient in the TaTME group needed reintervention by the Hartmann procedure, and the rest of the patients with anastomosis leaks were treated with antibiotics and parenteral nutrition. Based on meta-analysis, some authors ${ }^{26}$ have reported significant differences in relation to anastomotic leak rate in favour of TaTME group, but another systematic review ${ }^{25}$ could not demonstrate any differences, as we did in the present study.

One of the most critical steps during middle-/lower-third rectal cancer surgery is performing the colorectal or coloanal anastomosis. The main principles to achieve an optimal anastomosis during TaTME and LaTME are well-vascularizated ends, tension-free suture, and descending colon without twisting. The main difference between TaTME and LaTME regarding stapled colorectal anastomosis is the open rectal stump after the TaTME procedure. A full-thickness pursestring suture of the open stump is required. In cases of long distal rectal stumps, the purse-string suture could be performed through the transanal platform, and, in cases of short distal rectal stumps, the purse-string suture could be performed under direct visualization; therefore, it could result easier to surgeons. The more distal the colorectal anastomosis, the easier it is to perform the purse-string suture of the rectal stump during TaTME, and the more difficult it is to introduce and to shout stapling devices during laTME. ${ }^{7}$ Thus, we consider that TaTME may offer important technical advantages in cases of lower-third rectal cancer. However, the great technical advantage of this critical surgical step did not result in great significant benefits regarding the rate of anastomotic leak, at least in the present study.

In the present study, other quality indicators of rectal cancer surgery, such as the length of the hospital stay, and the rates of mortality, reoperation, and readmission, were similar in both groups. Other studies ${ }^{25,26}$ have reported lower rates of reoperation and readmission and shorter lengths of hospital stay after TaTME. We did not analyse the quality of life and functional outcomes after TaTME, but Koedman et al., ${ }^{29}$ in a prospective study including 33 patients, reported that TaTME was associated with acceptable quality of life and functional outcomes based on validated questionnaires.

During TaTME, the distal end of the tumor could be directly visualized through the transanal platform, so the distal margin of resection could be correctly chosen before starting the dissection under direct visualization longer than $1 \mathrm{~cm}$. This advantage could enable surgeons to preserve as much length of the rectum as possible, and avoid obtaining an uncertain DRM in case of unfavourable conditions for rectal surgery presented during LaTME. In the present series, we obtained a free DRM in all cases in the TaTME group. Two meta-analysis ${ }^{25,26}$ reported low rates of positive DRM in TaTME, but they could not demonstrate statistically significant differences between TaTME and laTME.

The principal aim of TME for rectal cancer surgery is to remove a clear CRM, because positive CRM is associated with significantly increased local and systemic recurrences rates. ${ }^{30}$ In the present study, we did not find statistically 
significant differences between the groups in terms of the quality of the TME and the rate of positive CRM. However a meta-analysis ${ }^{25}$ reported that the TaTME group had a significantly higher rates of complete specimens, longer CRM, and lower positive CRM involvement. In this sense, Veltcamp et al. ${ }^{31}$ demonstrated that the completeness of the TME was significantly better with TaTME than LaTME based on the identification of the residual mesorectum in postoperative MRI scans. Data from the international TaTME Registry ${ }^{32}$ including 2,653 patients with rectal cancer show a rate of $4 \%$ of positive CRM. The authors ${ }^{32}$ demonstrated that the predictive factors for positive CRM were tumors located up to $1 \mathrm{~cm}$ from the anorectal junction, anterior tumors, cT4 tumors, extramural venous invasion, and threatened or involved CRM on baseline MRI.

All of these favourable short-term oncological outcomes of TaTME could make surgeons optimistic about this novel surgical technique. However, in a Norwegian randomized clinical trial ${ }^{33}$ analyzing the long-term oncological outcomes of TaTME among 157 patients, the authors reported a higher local recurrence rate compared with the national rate. This adverse result led to the suspension of the TaTME program in Norway, and it is a warning for the community of surgeons to be cautious. Specific training programs for colorectal surgeons should be stablished, and the selection of patients who will undergo the TaTME procedure must be strictly rigorous.

There were some limitations to the present study: it describes the short-term outcomes of TaTME at our institution. Although both study groups were homogeneous in terms of the main clinical features, the present is not a randomized study, and weincluded few patients. Randomized controlled trials are needed, but, while we wait for the resuls of the COLOR III trial, the available outcomes of the International TaTME Registry ${ }^{27,28,32}$ and of meta-analyses suggest clinical advantages regarding the TaTME procedure.

In conclusion, the present study reports that the indicators of quality for rectal cancer surgery were similar in the TaTME and LaTME groups. In cases of lower-/middle third rectal cancer, TaTME is safe and feasible, and it could be a possible alternative to LaTME in unfavourable technical conditions. However, randomized clinical trials on the functional and oncological long-term outcomes are needed to evaluate this procedure as an alternative surgical technique for lower-third rectal cancer.

\section{References}

1 North AB, South CD. (2017). Cancer Incidence in Antarctica (20082012). In: Bray F, Colombet M, Mery L, Piñeros M, Znaor A, Zanetti R, Ferlay J, editors. Cancer Incidence in Five Continents. , Vol. XI (electronic version). Lyon: International Agency for Research on Cancer. Available from: http://ci5.iarc.fr, accessed [date] .

2 Lepage C, Bossard N, Dejardin O, Carmona-Garcia MC, Manfredi S, Faivre JGRELL EUROCARE-5 Working Group. Trends in net survival from rectal cancer in six European Latin countries: results from the SUDCAN population-based study. Eur J Cancer Prev 2017;26 Trends in cancer net survival in six European Latin Countries: the SUDCAN study:S48-S55
3 Heald RJ, Husband EM, Ryall RDH. The mesorectum in rectal cancer surgery-the clue to pelvic recurrence? Br J Surg 1982;69 (10):613-616

4 Fleshman J, Branda M, Sargent DJ, et al. Effect of LaparoscopicAssisted Resection vs Open Resection of Stage II or III Rectal Cancer on Pathologic Outcomes: The ACOSOG Z6051 Randomized Clinical Trial. JAMA 2015;314(13):1346-1355

5 Stevenson AR, Solomon MJ, Lumley JW, Hewett P, Clouston AD. gebski VJ, Davies L, Wilson K, Hague W, Simes J. Effect of Laparoscopic Assisted Resection vs Open Resection on Patological Outcomes in Rectal Cancer: The AlaCaRT Randomized Clinical Trial. JAMA 2015;314:1356-1363

6 Sylla P, Rattner DW, Delgado S, Lacy AM. NOTES transanal rectal cancer resection using transanal endoscopic microsurgery and laparoscopic assistance. Surg Endosc 2010;24(05):1205-1210

7 Labalde Martínez M, García Borda J, Nevado García C, Alcalde Escribano J, de la Cruz Vigo F, Ferrero Herrero E. Escisión mesorrectal total transanal (TaTME): Aspectos prácticos de la técnica quirúrgica. Rev Español Invest Quirúr 2019;4:147-155

8 Jessup J, Benson A, Chen V. Colon and Rectum. In: Amin MB, Edge SB, Greene FLet al., eds.: AJCC Cancer Staging Manual. $8^{\text {th }}$ ed. New York, NY: Springer; 2017:251-74

9 Nagtegaal ID, van de Velde CJH, van der Worp E, Kapiteijn E, Quirke P, van Krieken JHCooperative Clinical Investigators of the Dutch Colorectal Cancer Group. Macroscopic evaluation of rectal cancer resection specimen: clinical significance of the pathologist in quality control. J Clin Oncol 2002;20(07):1729-1734

10 Ryan R, Gibbons D, Hyland JM, et al. Pathological response following long-course neoadjuvant chemoradiotherapy for locally advanced rectal cancer. Histopathology 2005;47(02):141-146

11 Dindo D, Demartines N, Clavien PA. Classification of surgical complications: a new proposal with evaluation in a cohort of 6336 patients and results of a survey. Ann Surg 2004;240(02): 205-213

12 Wiegering A, Buhr HJ, Klinger C, et al. [Quality indicators for surgery of rectal cancer : Evidence-based development of a set of indicators for quality]. Chirurg 2018;89(01):26-31

13 Labalde Martinez M, Garcia Borda FJ, Alcalde J, et al. Transanal total mesorectal excision and adverse conditions for laparoscopic total excision. Eur Surg 2020;52:88-95. doi https://doi.org/ $10.1007 / /$ S10353-019-00626-Y

14 Akiyoshi T, Kuroyanagi H, Oya M, et al. Factors affecting the difficulty of laparoscopic total mesorectal excision with double stapling technique anastomosis for low rectal cancer. Surgery 2009;146(03):483-489

15 Jayne D, Pigazzi A, Marshall H, et al. Gudgeon Mn, Pietro Bianchi PP, Edlin R,Hulme C, Brown J Effect of robotic-assisted vs conventional laparoscopic surgery on risk of conversion to open laparotomy among patients undergoing resection for rectal cancer: the ROLARR randomized clinical trial. JAMA 2017;318(16):1569-1580

16 Sachdeva U, Sylla P. Natural orifice approaches in rectal surgery: transanal endoscopic protectomy. In: Pigazzi Aeditor. Techniques in minimallyt invasive rectal surgery. Basel, Switzerland: Springer; 2018:151-76

17 Westwood DA, Cuda TJ, Hamilton AER, Clark D, Stevenson ARL. Transanal total mesorectal excision for rectal cancer: state of the art. Tech Coloproctol 2018;22(09):649-655

18 Baker EJ, Water PS, Peacock O, McCormick JJ. Advanced application of TaTME platform for a T4 anterior rectal tumor. Surg Laparosc Percutan Tech; 2019

19 Yamaguchi T, Imai M, Uematsu D. Hybrid approach using laparoscopy and transanal minimally invasive surgery to treat rectal cancer with invasion to the seminal vesicles. Asian J Endosc Surg 2017;10(02):219-222

20 Uematsu D, Akiyama G, Sugihara T, et al. Transanal total pelvic exanteration with sphincter-preserving surgery. Dis Colon Rectum 2018;61(05):641 
21 Uematsu D, Akiyama G, Sugihara T, Magishi A, Yamaguchi T, Sano T. Transanal total pelvic exanteration: pushing the limits of transanal total mesorectal excision with transanal pelvic exanteration. Dis Colon Rectum 2017;60(06):647-648

22 D'Andrea AP, McLemore EC, Bonaccorso A, et al. Transanal total mesorectal excision (taTME) for rectal cancer: beyond the learning curve. Surg Endosc 2020;34(09):4101-4109

23 Lelong B, de Chaisemartin C, Meillat Het al;French Research Group of Rectal Cancer Surgery (GRECCAR). A multicenter randomized controlled trial to evaluate the efficacy, morbidity and functional outcome of endoscopic transanalprotectomy versus laparoscopic protectomy for low-lying rectal cancer (ETAP-GRECCAR 11 TRI$\mathrm{AL})$ : rationale and design. BMC Cancer 2017;17:253

24 Deijen CL, Velthuis S, Tsai A, Mavroveli S. de Lange-de KlerkESMk, Sietses C, Tuynman JB,Lacy AM, Hanna GB, Bonjer HJ. COLOR III: a multicenter randomized clinical trial comparing transanal TME versus laparoscopic TME for mid and low rectal cancer. Surg Endosc 2016;30:3210-3215

25 Ma B, Gao P, Song Y, et al. Transanal total mesorectal excision (taTME) for rectal cancer: a systematic review and meta-analysis of oncological and perioperative outcomes compared with laparoscopic total mesorectal excision. BMC Cancer 2016;16:380-393

26 Aubert M, Mege D, Panis Y. Total mesorectal excision for low and middle rectal cancer: laparoscopic versus transanal approach-a meta-analysis. Surg Endosc 2020;34(09):3908-3919

27 Penna M, Hompes R, Arnold S, et al; TaTME Registry Collaborative. TaTME registry collaborative. Transanal total mesorectal excision.
International registry results of first 720 cases. Ann Surg 2017; 266(01):111-117

28 Penna M, Hompes R, Arnold Set al;TaTME registry collaborative. Incidence and risk factors for anastomotic failure in 1594 patients treated by transanal total mesorectal excision. Ann Surg 2019;269 (04):700-711

29 Koedam TW, van Ramshorst GH, Deijen CL, et al. Transanal total mesorectal excision (TaTME) for rectal cancer: effects on patientreported quality of life and functional outcome. Tech Coloproctol 2017;21(01):25-33

30 Nagtegaal ID, Quirke P. What is the role for the circumferential margin in the modern treatment of rectal cancer? J Clin Oncol 2008;26(02):303-312

31 Veltcamp Helbach M, Koedam TWA, Knol JJ, et al. Residual mesorectum on postoperative magnetic resonance imaging following transanal total mesorectal excision (TaTME) and laparoscopic total mesorectal excision (LapTME) in rectal cancer. Surg Endosc 2019;33(01):94-102

32 Roodbeen SX, de Lacy FB, van Dieren S, et al; International TaTME Registry Collaborative. Predictive factors and risk model for positive circumferential resection margin rate after transanal total mesorectal excision in 2653 patients with rectal cancer. Ann Surg 2019;270(05):884-891

33 Wasmuth HH, Faerden AE, Myklebust TA, et al; Norwegian TaTME Collaborative Group, on behalf of the Norwegian Colorectal Cancer Group. Transanal total mesorectal excision for rectal cancer has been suspended in Norway. Br J Surg 2020;107(01):121-130 